\title{
Mechanical and Thermal Properties of Polymethyl Methacrylate-BN Nanotube Composites
}

\author{
C. Y. Zhi, ${ }^{1}$ Yoshio Bando, ${ }^{1}$ Wenlong L. Wang, ${ }^{1}$ Chengchun C. Tang, ${ }^{1}$ Hiroaki Kuwahara, ${ }^{2}$ and Dmitri Golberg ${ }^{1}$ \\ ${ }^{1}$ International Center for Materials Nanoarchitectonics (MANA), National Institute for Materials Science (NIMS), \\ Namiki 1-1, Tsukuba, Ibaraki 305-0044, Japan \\ ${ }^{2}$ Innovation Research Institute, Teijin Ltd., 2-1, Hinode-cho, Iwakuni, Yamaguchi 740-8511, Japan \\ Correspondence should be addressed to C. Y. Zhi, zhi.chunyi@nims.go.jp
}

Received 23 July 2008; Revised 18 October 2008; Accepted 6 November 2008

Recommended by Gong-Ru Lin

\begin{abstract}
Polymethyl methacrylate (PMMA)-BN nanotube (BNNT) composites were fabricated and their mechanical and thermal properties were analyzed. Using a $1 \mathrm{wt} . \%$ BNNTs fraction in a polymer, the elastic modulus of PMMA was increased up to $19 \%$. In addition, thermal stability and glass transition temperature of PMMA were also positively affected. The thermal conductivity of PMMA with BNNT additions increased three times. The resultant BNNT-PMMA composites possess the high electrical breakover voltages. Thus our studies clearly indicate that BNNTs are promising nanofillers for improvement of mechanical and thermal conductivity of dielectric polymers under preservation of their electrical insulation.
\end{abstract}

Copyright (c) 2008 C. Y. Zhi et al. This is an open access article distributed under the Creative Commons Attribution License, which permits unrestricted use, distribution, and reproduction in any medium, provided the original work is properly cited.

\section{INTRODUCTION}

The discovery of carbon nanotubes (CNTs) and observations of their unique, mechanical, thermal, and electrical properties have soon stimulated the studies on their usage in composites [1-3]. The composites with a matrix made of metal or ceramic have in fact been intensively studied, but the polymeric composite researches have become more widespread due to many comprehensive applications. For example, CNTs have been used to improve mechanical properties, thermal conductivity, and electrical conductance of polystyrene, polyacrylonitrile, polymethyl methacrylate (PMMA), and so forth [4-14]. However, there have been some unsolved problems with respect to real applications of CNTs-polymer composites, for example, nanotube dispersion at high concentrations. In addition, the mechanical performance of a CNT-polymer composite strongly depends on which kind of tubes, that is, arc-discharge-grown or catalytically produced, is in use. The arc-discharge CNTs possess excellent mechanical properties due to a hightemperature growth, but their production yield is typically low. Oppositely, a large amount of CNTs can be produced through a catalytic growth, but such tubes have plenty of structural defects, which is harmful for applications $[15,16]$.
Boron nitride nanotubes (BNNTs), which have very similar atomic structure to CNTs, exhibit a number of physical properties which could be advantageous (compared to CNTs) in polymeric composites. For example, a BNNT is a constant wide band gap material [17-19]. BNNTs could emit ultraviolet light under excitation by an electron beam. The theoretical estimates of the elastic modulus of a BNNT gave a value of $\sim 850 \mathrm{GPa},[20]$, that is, approximately 0.8 times of CNT. Moreover, BNNTs are chemically inert and structurally stable; they withstand heating in air up to $900^{\circ} \mathrm{C}$ [21]. It is worth noting that the thermal conductivity of BNNTs has been predicted to be even higher than that of CNTs $[22,23]$. All these factors make a BNNT useful as a filler in polymeric composites. We envisage that BNNTs can improve mechanical properties and thermal conductivity of a polymer while preserving its dielectric properties. Moreover, since BNNTs are pure white in color, the BNNTloaded polymers should keep good transparency at low fraction of BNNTs. However, the BNNT-polymer compositerelated studies have been overlooked in the literature. Only recently, the present authors have reported that BNNTs improve the tensile modulus of a polystyrene [24]. The detailed studies on other polymers and their properties have still been lacking. 
TABLE 1: Elastic modulus, strength and elongation of a blank PMMA, and its BNNTs' composites.

\begin{tabular}{lcccc}
\hline Materials & Thickness $(\mu \mathrm{m})$ & Modulus $(\mathrm{GPa})$ & Strength $(\mathrm{MPa})$ & Elongation $(\%)$ \\
\hline PMMA & 25 & 2.07 & 58.31 & 9.91 \\
PMMA; 1 wt.\% BNNT & 27 & 2.47 & 54.35 & 3.93 \\
PMMA; 1 wt.\% BNNT & 52 & 2.51 & 54.73 & 4.21 \\
\hline
\end{tabular}

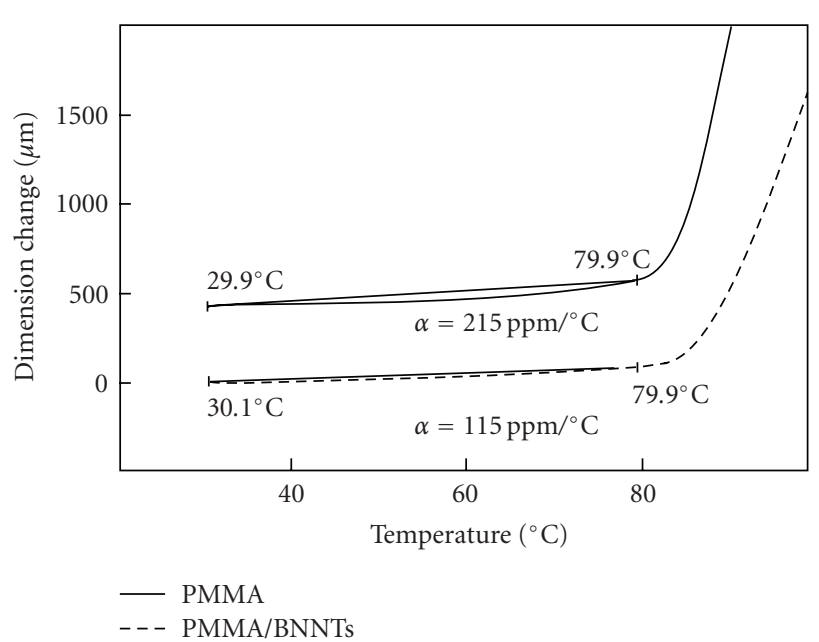

FIgURE 1: TMA of a blank PMMA and BNNTs/PMMA composites; the CTE is significantly reduced after adding BNNTs.

In this work, utilizing large quantities of highly pure BNNTs synthesized via a chemical vapor deposition method using boron powder and metal oxide as reactants (BOCVD), PMMA-BNNTs composites were fabricated using a solution method. Then the expected mechanical reinforcement and thermal conductivity improvement of the polymers were indeed been verified [25].

\section{EXPERIMENTAL DETAILS}

Tens of grams quantities of highly pure BNNTs were synthesized using the BOCVD method [25-28]. The asgrown BNNTs were heated up to $1900^{\circ} \mathrm{C}$ under protection of Ar gas in order to remove catalysts and other impurities. Scanning electron microscopy (SEM) was used to investigate the tube morphology, and electron energy loss (EELS) and energy dispersive X-ray (EDX) spectroscopes were used to confirm the absence of foreign species.

In order to fabricate PMMA-BNNT composites, $10 \mathrm{mg}$

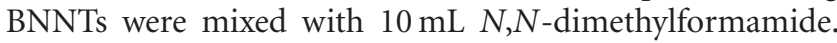
The mixture was magnetically stirred for 5 hours and sonicated over 100 minutes and a BNNT solution was obtained. Then, 990 mg PMMA was dissolved in the solution, followed by stirring and sonication. The obtained mixture was cast onto a glass plate. The doctor blades with $200 \mu \mathrm{m}$ and $400 \mu \mathrm{m}$ slit widths were used for casting of the resultant composite films. After heating to $60^{\circ} \mathrm{C}$ and solvent evaporation, the composite films were peeled off from the glass plates, and mounted on a frame for the complete removal of solvent.
The dried films were cut into $9 \times 1 \mathrm{~cm}$ slices for the exsitu tensile tests on an RTC 1225A apparatus (Orientec Inc., Tokyo, Japan). The slices were stretched until they broke. All experiments were performed at room temperature at a deformation rate of $5 \mathrm{~mm} / \mathrm{min}$.

Thermogravimetry (TGA) experiments were run on a Thermoplus TG8120 machine (Rigaku Inc., Tokyo, Japan). For differential scanning calorimetry (DSC), an SII Exstar6000 DSC6220 apparatus (Seiko Instruments. Inc., Tokyo, Japan) was utilized. The curves were recorded for the second heating cycle. Thermal mechanical analysis (TMA) was performed on a universal $\mathrm{V} 2.5 \mathrm{H}$ TA instrument using the sample slices with a length of around $1 \mathrm{~cm}$.

\section{RESULTS AND DISCUSSION}

The tensile mechanical properties of BNNT/PMMA composite films are summarized in Table 1 . With a $1 \mathrm{wt} \%$ BNNTs fraction, the elastic modulus of PMMA was improved by $19 \%$, but the tensile strength slightly decreased. No behavioral variations were observed for the different film thicknesses, as shown in Table 1. The slit widths of the doctor blades used were $200 \mu \mathrm{m}$ and $400 \mu \mathrm{m}$, thus much larger than the BNNTs lengths, and it is thought that a change in slit width should not affect the arrangement of BNNTs in a polymer matrix. It is worth noting that the film's thicknesses significantly decrease after solvent evaporation. These results show that the external force can be transferred to BNNTs in some degree, which results in an increase in elastic modulus; while the defects induced by BNNTs are still a problem, which leads to a decrease of strength. In all cases, the addition of fillers induces a dramatic reduction in elongation, which also indicates that the interaction between BNNTs and polymer chains exists.

For comparison, it is worth mentioning some related results on CNT/PMMA composites. W.-J. Lee et al. fabricated an MWCNT/PMMA nanocomposite by using both the injection molding and film casting processes [29]. The tensile strength of an MWCNT/PMMA nanocomposite increased by more than $15 \%$ and the tensile toughness increased by $\sim 17.5 \%$, compared to the blank PMMA. It is well known that surface modification can improve the nanotube dispersion and filler/matrix interfacial bonding. Recently, Liu and Wagner have used MWCNTs functionalized through covalent linking of long alkyl chains and prepared PMMA/CNT composites [30]. A 32\% rise in tensile modulus and a $28 \%$ increase in tensile strength were observed for a composite loaded with a $1.0 \mathrm{wt}$ \% fraction of CNT fillers. Keeping in mind that no surface functionalization and/or surfactants 


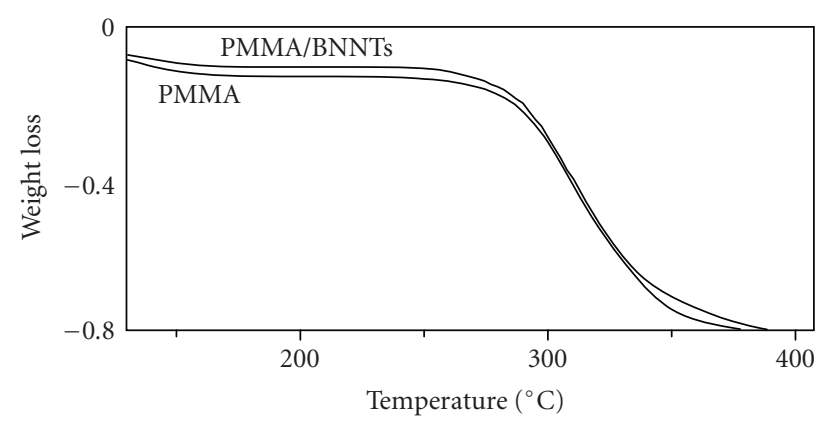

(a)

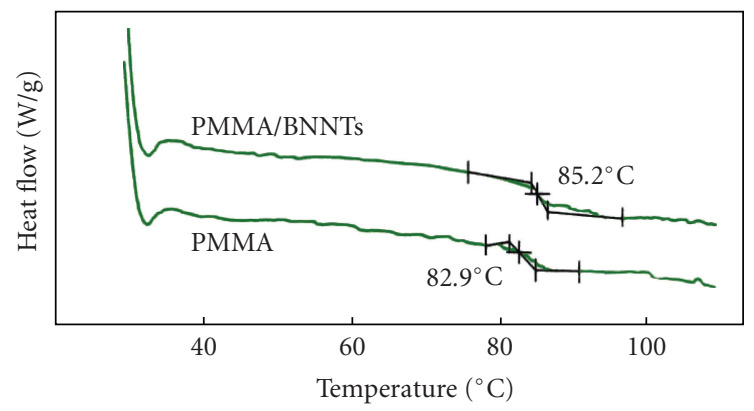

(b)

FIgure 2: (a) DSC and (b) TGA of a blank PMMA and BNNTs/PMMA composites, which indicate that the thermal stability and glass transition temperature are only slightly modified.

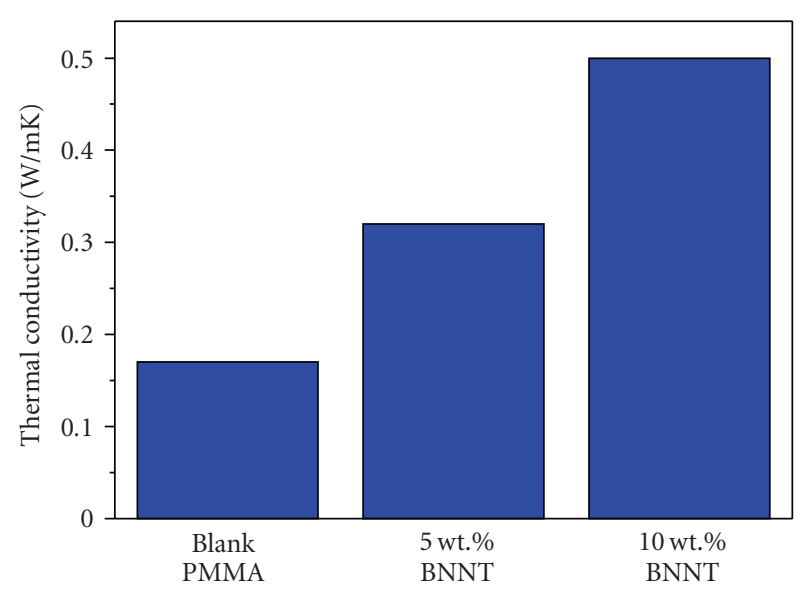

FIgURE 3: Thermal conductivity of a blank PMMA and BNNTs/ PMMA composites, almost 3-fold improvement was obtained with a $10 \mathrm{wt} . \%$ BNNT loading fraction.

were used in our experiments, our results are considered to rival this literature data.

In most of previous experiments related to CNT/polymer composites catalytically grown CNTs were utilized. However, the catalytically produced CNTs are not structurally perfect and are prone to defects. By contrast, the present BNNTs grown via the BOCVD method are well structured and possess a high elastic modulus of $500 \sim 700 \mathrm{GPa}$, as was documented using the in situ TEM techniques [31]. We note that it is rather difficult to directly compare the preexisting CNTs and the present BNNTs impacts since very different numbers have been reported for the CNT-loaded composites.

Since we did not use any special pretreatments of the BNNTs and special techniques for composite film fabrication it is envisaged here that there is still a plenty of room for further modulus improvement. In this respect, an estimate was performed to elucidate the theoretical mechanical properties of BNNTs/PMMA composites. Ideally, if BNNTs strongly bond to PMMA, the external tensile load will be transmitted from a matrix to BNNTs through the interfacial shear stress. Thus, a BNNTs/PMMA composite film can be considered as a randomly oriented discontinuous fiber lamina and the composite modulus, $E_{c}$, can be estimated using the following equations [32]:

$$
\begin{aligned}
& E_{C}=\left[\frac{3}{8} \frac{1+2\left(l_{N T} / d_{N T}\right) \eta_{L} V_{N T}}{1-\eta_{L} V_{N T}}+\frac{5}{8} \frac{1+2 \eta_{T} V_{N T}}{1-\eta_{T} V_{N T}}\right] E_{\mathrm{pmma}} \\
& \eta_{L}=\frac{\left(E_{N T} / E_{\mathrm{pmma}}\right)-1}{\left(E_{N T} / E_{\mathrm{pmma}}\right)+2\left(l_{N T} / d_{N T}\right)}, \\
& \eta_{T}=\frac{\left(E_{N T} / E_{\mathrm{pmma}}\right)-1}{\left(E_{N T} / E_{\mathrm{pmma}}\right)+2}
\end{aligned}
$$

where $E$ is the modulus, $l_{N T}$ is the length, and $d_{N T}$ is the outer diameter of BNNTs. The volume fraction, $V_{N T}$, is estimated to be $\sim 0.70$ vol. $\%$ for a $1 \mathrm{wt} . \%$ BNNT fraction. For the sonicated BNNTs used in our experiments, the average diameter and length are $50 \mathrm{~nm}$ and $\sim 6 \mu \mathrm{m}$, respectively. Thus $E_{c}$ was calculated to be $1.35 E_{\text {PMMA }}$ for a composite with a $1 \mathrm{wt} . \%$ BNNTs loading fraction. By taking a $3 \mathrm{wt} . \%$ BNNTs fraction the modulus of $1.98 E_{\text {PMMA }}$ was obtained. These estimates confirm that BNNTs are indeed very promising nanomaterials for the mechanical enhancement of polymeric composites. It is also predicted that, experimentally, notably better reinforcement can be achieved for covalently modified BNNTs.

The coefficient of thermal expansion (CTE) of composite films is shown in Figure 1. After adding BNNTs, the CTE of PMMA dramatically decreases from 215 to $115 \mathrm{ppm} /{ }^{\circ} \mathrm{C}$, that indicates that BNNTs significantly restrict the mobility of polymer chains. This is consistent with the above-discussed variations in the reinforced PMMA film elasticity and toughness.

TGA data is presented in Figure 2(a). The oxidation of the composites was characterized by the parameter $T_{0.1}$, which is the temperature corresponding to a $10 \%$ weight loss rate. $T_{0.1}$ is slightly changed when a $1 \mathrm{wt} . \%$ BNNTs fraction is in use. In line with our previous studies, it is suggested that BNNTs can in fact improve stability to oxidation. However, the effect is much smaller than in case of conventional phenolic antioxidants [33]. Such results are reasonable: on one hand, there are some localized states, such as vacancies, 
TABLE 2: Electrical breakover voltages of a blank PMMA and its BNNTs' composites.

\begin{tabular}{lccc}
\hline Materials & Thickness $(\mu \mathrm{m})$ & Volts applied $(\mathrm{V})$ & $\begin{array}{c}\text { Breakover } \\
\text { electric } \\
\text { field }(\mathrm{V} / \mu \mathrm{m})\end{array}$ \\
\hline $\begin{array}{l}\text { PMMA } \\
\text { PMMA; }\end{array}$ & 25 & 263 & 10.52 \\
5 wt.\% & 30 & 324 & 10.80 \\
$\begin{array}{l}\text { BNNT } \\
\text { PMMA; }\end{array}$ & & & \\
10 wt.\% & 34 & 312 & 9.17 \\
BNNT & & & \\
\hline
\end{tabular}

dangling bonds, and functional groups on BNNT surfaces which may be responsible for radical termination during polymer degradation. On the other hand, the effect only takes place on the interfaces between BNNTs and polymers, and the concentration of such lattice defects in BNNTs is low. These factors result in only marginal improvement of the oxidation resistance of PMMA and thus make doubtful an expected design of the fireproofing polymers out of such composites.

Blank PMMA has a glass transition temperature $T_{g}$ of $82.9^{\circ} \mathrm{C}$, as shown on a DSC curve in Figure 2(b). The $T_{g}$ is estimated from the midpoint of a heat capacity peak. The $T_{g}$ of a PMMA/BNNT composite becomes $\sim 85.2^{\circ} \mathrm{C}$. In case of organic-inorganic nanocomposites, the mobility of polymer chains is significantly affected by the confinement and strength of polymer-surface interactions. This applies to the interactions between BNNTs and PMMA chains. The chains which are in contact with BNNTs would exhibit slower dynamics than those within a polymer matrix. Actually, even in the absence of particularly favorable interactions, the contact with a hard BNNTs' surface would tend to increase the local segment density profile. This, in turn, leads to an increase in the configuration entropy, and thus an increase in $T_{g}$ looks reasonable. It is worth mentioning that the similar phenomenon was observed in many inorganicorganic composite systems [34].

Composite films with 5 wt.\% and 10 wt.\% BNNTs fractions were chosen for the thermal conductivity measurements. The results are shown in Figure 3. With a $10 \mathrm{wt} . \%$ BNNTs fraction, the thermal conductivity of PMMA increases from 0.17 to $0.50 \mathrm{~W} / \mathrm{mK}$ (nearly 3 -fold). It should be emphasized that this gain is likely to display the lower estimate for the observed improvement since the BNNT texture within the film is generally misaligned with the direction used for the heat flow measurements (because of experimental constraints) $[35,36]$.

The appealing point of BNNT usage in polymeric composites is that the original dielectric nature of a polymer is kept in the resultant composite. This fact is crucial in many cases, such as packing materials for electrical circuits, power modules, and so forth. Needless to say, carbon nanotubefilled polymers are not suitable for these purposes due to high electrical conductivity. In Table 2, the electrical breakover voltages of a blank PMMA and its composites are compared. This reveals that both blank PMMA and its BNNTs composites have a similar breakover electric field of $\sim 10 \mathrm{~V} / \mu \mathrm{m}$. Therefore, the presently developed BNNTs/polymer composites are surely suitable materials for heat-releasing parts due to unique combination of decent thermal conductivity and perfect electrical insulation.

\section{CONCLUSION}

In summary, BNNTs/PMMA composites were fabricated and their properties were elucidated. The elastic modulus of PMMA was improved up to $19 \%$ while using only a $1 \mathrm{wt} . \%$ BNNTs loading fraction. The coefficient of thermal expansion of a PMMA film dramatically reduced due to profound interactions between BNNTs and PMMA chains. This fact was consistent with the corresponding variations in glass transition temperatures. Thermal conductivity of PMMA loaded with a $10 \mathrm{wt} . \%$ BNNT fraction was improved 3 times compared to blank PMMA. The developed BNNT/PMMA composite films simultaneously possessing (i) improved thermal conductivity, (ii) enhanced mechanical properties, and (iii) perfect electrical insulation are envisaged to find diverse applications in many polymeric fields.

\section{ACKNOWLEDGMENTS}

The authors thank Dr. H. Sakurai, Dr. Y. Uemura, Dr. M. Mitome, and Dr. K. Kurashima for their cooperation and kind help. This work was in part supported by World Premier International Research Center (WPI) Initiative on Materials Nanoarchitectonics, MEXT, Japan.

\section{REFERENCES}

[1] S. Iijima, "Helical microtubules of graphitic carbon," Nature, vol. 354, no. 6348, pp. 56-58, 1991.

[2] R. Andrews and M. C. Weisenberger, "Carbon nanotube polymer composites," Current Opinion in Solid State and Materials Science, vol. 8, no. 1, pp. 31-37, 2004.

[3] P. J. F. Harris, "Carbon nanotube composites," International Materials Reviews, vol. 49, no. 1, pp. 31-43, 2004.

[4] M.-F. Yu, B. S. Files, S. Arepalli, and R. S. Ruoff, "Tensile loading of ropes of single wall carbon nanotubes and their mechanical properties," Physical Review Letters, vol. 84, no. 24, pp. 5552-5555, 2000.

[5] M.-F. Yu, O. Lourie, M. J. Dyer, K. Moloni, T. F. Kelly, and R. S. Ruoff, "Strength and breaking mechanism of multiwalled carbon nanotubes under tensile load," Science, vol. 287, no. 5453, pp. 637-640, 2000.

[6] M. C. Weisenberger, E. A. Grulke, D. Jacques, T. Rantell, and R. Andrews, "Enhanced mechanical properties of polyacrylonitrile/multiwall carbon nanotube composite fibers," Journal of Nanoscience and Nanotechnology, vol. 3, no. 6, pp. 535-539, 2003.

[7] D. Qian, E. C. Dickey, R. Andrews, and T. Rantell, "Load transfer and deformation mechanisms in carbon nanotubepolystyrene composites," Applied Physics Letters, vol. 76, no. 20, pp. 2868-2870, 2000.

[8] E. T. Thostenson and T.-W. Chou, "Aligned multi-walled carbon nanotube-reinforced composites: processing and 
mechanical characterization," Journal of Physics D, vol. 35, no. 16, pp. L77-L80, 2002.

[9] A. B. Dalton, S. Collins, E. Muñoz, et al., "Super-tough carbon-nanotube fibres," Nature, vol. 423, no. 6941, p. 703, 2003.

[10] A. Eitan, K. Jiang, D. Dukes, R. Andrews, and L. S. Schadler, "Surface modification of multiwalled carbon nanotubes: toward the tailoring of the interface in polymer composites," Chemistry of Materials, vol. 15, no. 16, pp. 3198-3201, 2003.

[11] L. S. Schadler, S. C. Giannaris, and P. M. Ajayan, "Load transfer in carbon nanotube epoxy composites," Applied Physics Letters, vol. 73, no. 26, pp. 3842-3844, 1998.

[12] P. M. Ajayan, L. S. Schadler, C. Giannaris, and A. Rubio, "Single-walled carbon nanotube-polymer composites: strength and weakness," Advanced Materials, vol. 12, no. 10, pp. 750-753, 2000.

[13] A. H. Barber, S. R. Cohen, and H. D. Wagner, "Measurement of carbon nanotube-polymer interfacial strength," Applied Physics Letters, vol. 82, no. 23, pp. 4140-4142, 2003.

[14] H. D. Wagner, O. Lourie, Y. Feldman, and R. Tenne, "Stressinduced fragmentation of multiwall carbon nanotubes in a polymer matrix," Applied Physics Letters, vol. 72, no. 2, pp. 188-190, 1998.

[15] E. Hernández, C. Goze, P. Bernier, and A. Rubio, "Elastic properties of $\mathrm{C}$ and $B_{x} C_{y} N_{z}$ composite nanotubes," Physical Review Letters, vol. 80, no. 20, pp. 4502-4505, 1998.

[16] J.-P. Salvetat, A. J. Kulik, J.-M. Bonard, et al., "Elastic modulus of ordered and disordered multiwalled carbon nanotubes," Advanced Materials, vol. 11, no. 2, pp. 161-165, 1999.

[17] X. Blase, A. Rubio, S. G. Louie, and M. L. Cohen, "Stability and band gap constancy of boron nitride nanotubes," Europhysics Letters, vol. 28, no. 5, pp. 335-340, 1994.

[18] X. D. Bai, E. G. Wang, J. Yu, and H. Yang, "Blue-violet photoluminescence from large-scale highly aligned boron carbonitride nanofibers," Applied Physics Letters, vol. 77, no. 1, pp. 67-69, 2000.

[19] J. Yu, J. Ahn, S. F. Yoon, et al., "Semiconducting boron carbonitride nanostructures: nanotubes and nanofibers," Applied Physics Letters, vol. 77, no. 13, pp. 1949-1951, 2000.

[20] A. P. Suryavanshi, M.-F. Yu, J. Wen, C. Tang, and Y. Bando, "Elastic modulus and resonance behavior of boron nitride nanotubes," Applied Physics Letters, vol. 84, no. 14, pp. 25272529, 2004.

[21] D. Golberg, Y. Bando, K. Kurashima, and T. Sato, "Synthesis and characterization of ropes made of BN multiwalled nanotubes," Scripta Materialia, vol. 44, no. 8-9, pp. 1561-1565, 2001.

[22] P. Kim, L. Shi, A. Majumdar, and P. L. McEuen, "Thermal transport measurements of individual multiwalled nanotubes," Physical Review Letters, vol. 87, no. 21, Article ID 215502, 4 pages, 2001.

[23] Y. Xiao, X. H. Yan, J. X. Cao, J. W. Ding, Y. L. Mao, and J. Xiang, "Specific heat and quantized thermal conductance of singlewalled boron nitride nanotubes," Physical Review B, vol. 69, no. 20, Article ID 205415, 5 pages, 2004.

[24] C. Zhi, Y. Bando, C. Tang, S. Honda, H. Kuwahara, and D. Golberg, "Boron nitride nanotubes/polystyrene composites," Journal of Materials Research, vol. 21, no. 11, pp. 2794-2800, 2006.

[25] C. Zhi, Y. Bando, G. Shen, C. Tang, and D. Golberg, "Boron nitride nanotubes: nanoparticles functionalization and junction fabrication," Journal of Nanoscience and Nanotechnology, vol. 7, no. 2, pp. 530-534, 2007.
[26] W. Han, Y. Bando, K. Kurashima, and T. Sato, "Synthesis of boron nitride nanotubes from carbon nanotubes by a substitution reaction," Applied Physics Letters, vol. 73, no. 21, pp. 3085-3087, 1998.

[27] C. Tang, Y. Bando, T. Sato, and K. Kurashima, "A novel precursor for synthesis of pure boron nitride nanotubes," Chemical Communications, no. 12, pp. 1290-1291, 2002.

[28] C. Zhi, Y. Bando, C. Tan, and D. Golberg, "Effective precursor for high yield synthesis of pure BN nanotubes," Solid State Communications, vol. 135, no. 1-2, pp. 67-70, 2005.

[29] W.-J. Lee, S.-E. Lee, and C.-G. Kim, "The mechanical properties of MWNT/PMMA nanocomposites fabricated by modified injection molding," Composite Structures, vol. 76, no. 4, pp. 406-410, 2006.

[30] L.-Q. Liu and H. D. Wagner, "A comparison of the mechanical strength and stiffness of MWNT-PMMA and MWNT-epoxy nanocomposites," Composite Interfaces, vol. 14, no. 4, pp. 285297, 2007.

[31] D. Golberg, P. M. F. J. Costa, O. Lourie, et al., "Direct force measurements and kinking under elastic deformation of individual multiwalled boron nitride nanotubes," Nano Letters, vol. 7, no. 7, pp. 2146-2151, 2007.

[32] P. K. Mallick, Fiber-Reinforced Composites, Marcel Dekker, New York, NY, USA, 1993.

[33] P. C. P. Watts, P. K. Fearon, W. K. Hsu, N. C. Billingham, H. W. Kroto, and D. R. M. Walton, "Carbon nanotubes as polymer antioxidants," Journal of Materials Chemistry, vol. 13, no. 3, pp. 491-495, 2003.

[34] J. Q. Pham, C. A. Mitchell, J. L. Bahr, J. M. Tour, K. Krishanamoorti, and P. F. Green, "Glass transition of polymer/single-walled carbon nanotube composite films," Journal of Polymer Science Part B, vol. 41, no. 24, pp. 33393345, 2003.

[35] Y. Xu and K. Yagi, "Calculation of the thermal conductivity of randomly dispersed composites using a finite element modeling method," Materials Transactions, vol. 45, no. 8, pp. 2602-2605, 2004.

[36] Y. Xu, M. Yamazaki, H. Wang, and K. Yagi, "Development of an internet system for composite design and thermophysical property prediction," Materials Transactions, vol. 47, no. 8, pp. 1882-1885, 2006. 

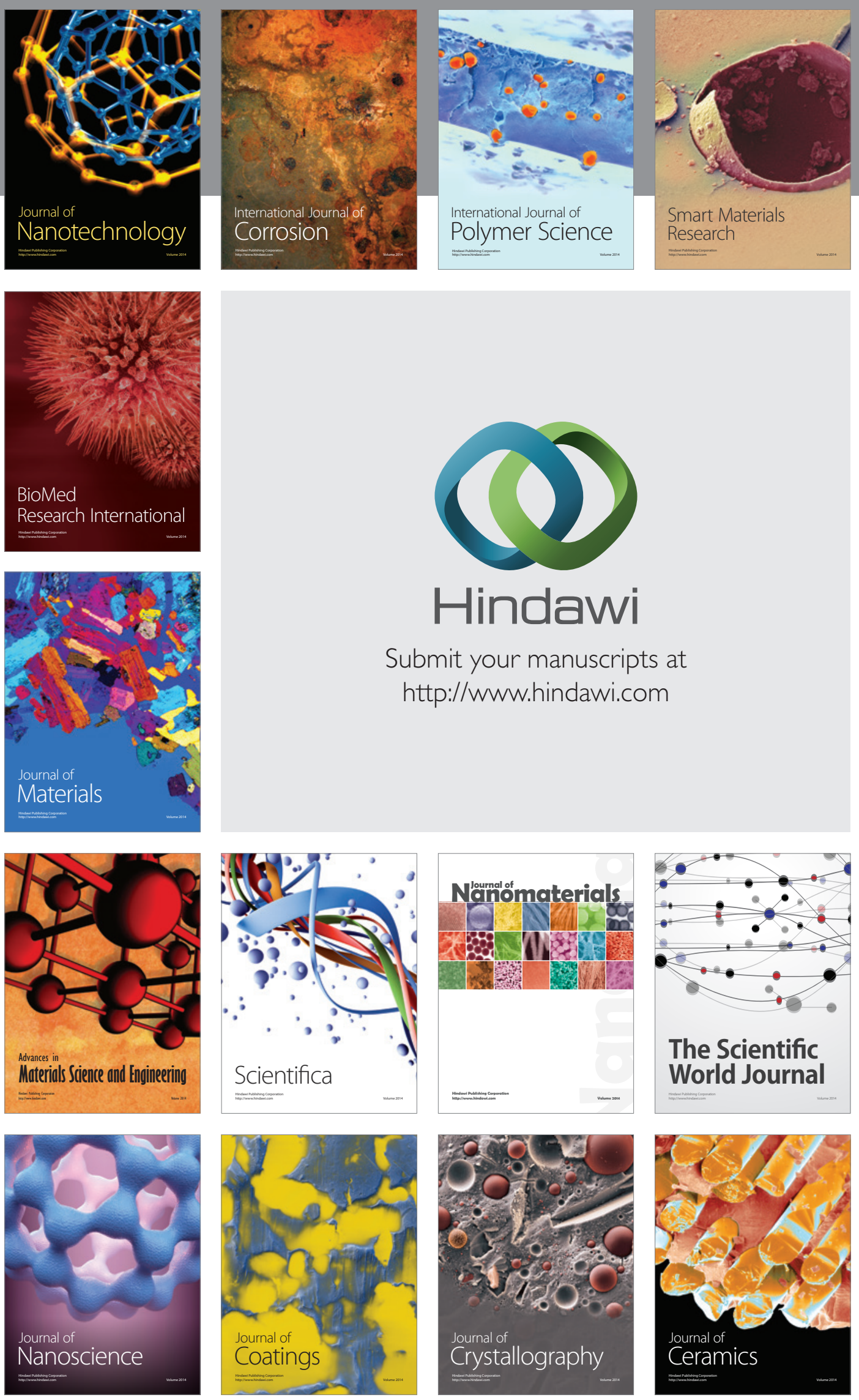

The Scientific World Journal

Submit your manuscripts at

http://www.hindawi.com

\section{World Journal}

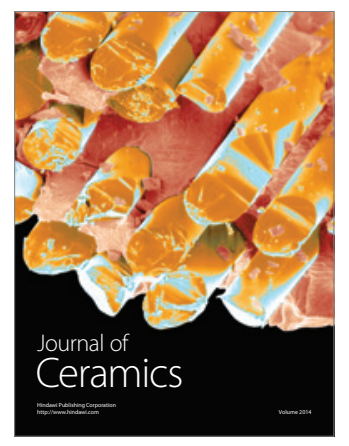

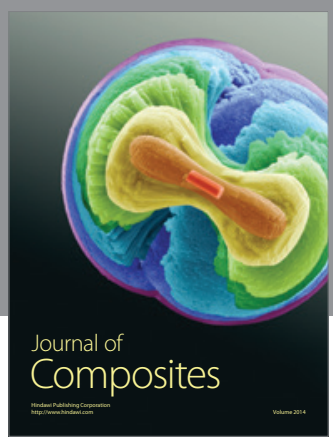
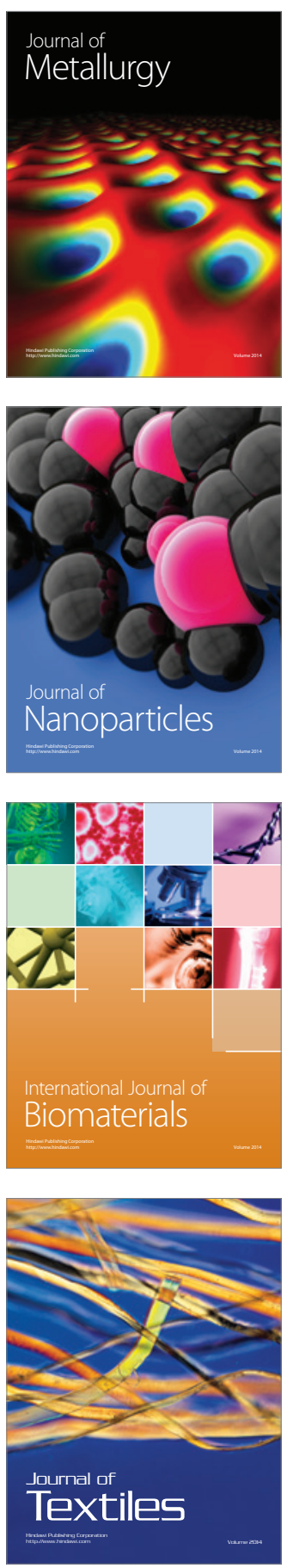